Acta Crystallographica Section E

Structure Reports

Online

ISSN 1600-5368

\title{
5-Fluoro-6' $\boldsymbol{H}, \boldsymbol{7}^{\prime} \boldsymbol{H}, \mathbf{8}^{\prime} \boldsymbol{H}$-spiro[indoline-3, $\mathbf{7}^{\prime}$ - pyrano[3,2-c:5,6-c']di-1-benzopyran]- $2,6^{\prime}, 8^{\prime}$-trione
}

\section{Abdulrahman I. Almansour, ${ }^{a}$ Raju Suresh Kumar, ${ }^{a}$ Natarajan Arumugam, ${ }^{a}$ P. Devi Shree ${ }^{b}$ and J. Suresh ${ }^{b *}$}

a Department of Chemistry, College of Sciences, King Saud University, PO Box 2455, Riyadh 11451, Saudi Arabia, and ${ }^{\mathbf{b}}$ Department of Physics, Madura College, Madurai 625011 , India

Correspondence e-mail: ambujasureshj@yahoo.com

Received 5 February 2012; accepted 13 February 2012

Key indicators: single-crystal X-ray study; $T=293 \mathrm{~K}$; mean $\sigma(\mathrm{C}-\mathrm{C})=0.002 \AA$; $R$ factor $=0.045 ; w R$ factor $=0.124 ;$ data-to-parameter ratio $=17.7$.

In the title compound, $\mathrm{C}_{26} \mathrm{H}_{12} \mathrm{FNO}_{6}$, the central pyran ring and both benzopyran systems are nonplanar, having total puckering amplitudes of 0.139 (2), 0.050 (1) and 0.112 (2) A, respectively. The central pyran ring adopts a boat conformation. The crystal structure is stabilized by $\mathrm{C}-\mathrm{H} \cdots \mathrm{O}, \mathrm{N}-$ $\mathrm{H} \cdots \mathrm{O}, \mathrm{N}-\mathrm{H} \cdots \mathrm{F}$ and $\mathrm{C}-\mathrm{H} \cdots \pi$ interactions.

\section{Related literature}

For the background to benzopyran derivatives, see: Martin \& Critchlow (1999); Teague \& Davis (1999); Joshi \& Jain (1985); Ninamiya (1980); Kobayashi \& Matsuda (1970). For hydrogen-bonding motifs, see: Bernstein et al. (1995).<smiles>O=C1Nc2ccc(F)cc2C12c1c(c3ccccc3oc1=O)Oc1c2c(=O)oc2ccccc12</smiles>

\section{Experimental}

Crystal data

$\mathrm{C}_{26} \mathrm{H}_{12} \mathrm{FNO}_{6}$

$M_{r}=453.37$

Triclinic, $P \overline{1}$

$a=7.8262$ (1) ^

$b=10.9278(1) \AA$

$c=12.4067$ (2) $\AA$

$\alpha=113.374(1)^{\circ}$

$\beta=94.922(1)^{\circ}$

\section{Data collection}

Bruker Kappa APEXII diffractometer

Absorption correction: multi-scan (SADABS; Sheldrick, 1996)

$T_{\min }=0.973, T_{\max }=0.978$

Refinement

$R\left[F^{2}>2 \sigma\left(F^{2}\right)\right]=0.045$

$w R\left(F^{2}\right)=0.124$

$S=1.04$

5504 reflections

311 parameters

1 restraint

Table 1

Hydrogen-bond geometry $\left(\AA{ }^{\circ}\right)$.

$C g 1$ is the centroid of the $\mathrm{C} 41-\mathrm{C} 46$ ring.

\begin{tabular}{|c|c|c|c|c|}
\hline$D-\mathrm{H} \cdots A$ & $D-\mathrm{H}$ & $\mathrm{H} \cdots A$ & $D \cdots A$ & $D-\mathrm{H} \cdots A$ \\
\hline $\mathrm{C} 45-\mathrm{H} 45 \cdots \mathrm{O} 5^{\mathrm{i}}$ & 0.93 & 2.50 & $3.2564(18)$ & 139 \\
\hline $\mathrm{C} 22-\mathrm{H} 22 \cdots{ }^{\mathrm{ii}}$ & 0.93 & 2.46 & $3.2205(17)$ & 139 \\
\hline $\mathrm{C} 66-\mathrm{H} 66 \cdots \mathrm{O}^{\mathrm{ii}}$ & 0.93 & 2.50 & $3.2921(17)$ & 144 \\
\hline $\mathrm{C} 64-\mathrm{H} 64 \cdots \mathrm{O} 3^{\mathrm{iii}}$ & 0.93 & 2.49 & 3.2641 (18) & 141 \\
\hline $\mathrm{C} 25-\mathrm{H} 25 \cdots C g 1^{\text {iv }}$ & 0.93 & 2.65 & 3.5482 (17) & 163 \\
\hline $\mathrm{N} 1-\mathrm{H} 1 \cdots \mathrm{F} 1^{\mathrm{v}}$ & $0.86(2)$ & $2.15(2)$ & $2.810(2)$ & $134(2)$ \\
\hline $\mathrm{N} 1-\mathrm{H} 1 \cdots \mathrm{O} 5^{\mathrm{i}}$ & $0.86(2)$ & $2.51(2)$ & $3.208(2)$ & $140(2)$ \\
\hline
\end{tabular}

Symmetry codes: (i) $-x,-y+1,-z+1$; (ii) $-x,-y+1,-z$; (iii) $x+1, y+1, z$; (iv) $-x,-y,-z ;($ v) $x-1, y, z$.

Data collection: APEX2 (Bruker, 2004); cell refinement: SAINT (Bruker, 2004); data reduction: SAINT; program(s) used to solve structure: SHELXS97 (Sheldrick, 2008); program(s) used to refine structure: SHELXL97 (Sheldrick, 2008); molecular graphics: PLATON (Spek, 2009); software used to prepare material for publication: SHELXL97.

This project was supported by the Research Center, Deanship of Scientific Research, College of Science, King Saud University.

Supplementary data and figures for this paper are available from the IUCr electronic archives (Reference: TK5055).

\section{References}

Bernstein, J., Davis, R. E., Shimoni, L. \& Chang, N.-L. (1995). Angew. Chem. Int. Ed. Engl. 34, 1555-1573.

Bruker (2004). APEX2 and SAINT. Bruker AXS Inc., Madison, Wisconsin, USA.

Joshi, K. C. \& Jain, R. (1985). Heterocycles, 23, 957-996.

Kobayashi, G. \& Matsuda, Y. (1970). Jpn Patent No. 7025894.

Martin, E. J. \& Critchlow, R. E. (1999). J. Combin. Chem. 1, 32-45.

Ninamiya, K. (1980). Jpn Patent No. 80164683.

Sheldrick, G. M. (1996). SADABS. University of Göttingen, Germany.

Sheldrick, G. M. (2008). Acta Cryst. A64, 112-122.

Spek, A. L. (2009). Acta Cryst. D65, 148-155.

Teague, S. J. \& Davis, A. M. (1999). Angew. Chem. Int. Ed. 38, 3743-3748.
5504 independent reflections 4486 reflections with $I>2 \sigma(I)$ independent and constrained

$\Delta \rho_{\min }=-0.29{\mathrm{e} \AA^{-3}}^{-3}$ $\rho_{\max }=0.46$ e $\AA^{-3}$ $\gamma=100.295(1)^{\circ}$

$V=943.77(2) \AA^{3}$

$Z=2$

Mo $K \alpha$ radiation

$\mu=0.12 \mathrm{~mm}^{-1}$

$T=293 \mathrm{~K}$

$0.23 \times 0.21 \times 0.18 \mathrm{~mm}$ 


\title{
supporting information
}

Acta Cryst. (2012). E68, o744 [doi:10.1107/S1600536812006332]

\section{5-Fluoro-6' $H, 7^{\prime} H, 8^{\prime} H$-spiro[indoline-3,7'-pyrano[3,2-c:5,6-c'] di-1-benzo- pyran]-2,6', 8'-trione}

\author{
Abdulrahman I. Almansour, Raju Suresh Kumar, Natarajan Arumugam, P. Devi Shree and J. \\ Suresh
}

\section{S1. Comment}

Benzopyran is a structural motif observed in many biologically active natural products and it plays an important role in binding to various biopolymers (Martin \& Critchlow, 1999; Teague \& Davis, 1999). Spiro indoles are also known for their broad spectrum of biological activities (Joshi \& Jain, 1985). Of the various spiro indoles, the spiro[indole-pyran] system has attracted attention owing to its interesting pharmacological properties (Ninamiya, 1980; Kobayashi \& Matsuda, 1970). The biological importance of these heterocycles in conjunction with our research interests, prompted us to synthesize and report the X-ray structure of the title compound, (I).

In (I), Fig. 1, the central pyrano ring A (O1-C6) and both the benzopyran rings B (C5/C6/C61-C66/O4/C51), C (C3/C3/C21-C26/O2/C31) are non-planar, having total puckering amplitudes, $\mathrm{Q}_{\mathrm{T}}$, of 0.139 (2), 0.050 (1) and 0.112 (2) $\AA$, respectively. The central pyrano ring adopts a boat conformation $\left[\Phi=357.6(6)^{\circ}\right.$ and $\left.\theta=109.0(6)^{\circ}\right]$. In the indolin-2-one system, the benzene and pyrrole rings are individually planar and make a dihedral angle of $2.20(1)^{\circ}$. The indoline-2-one system is in a perpendicular configuration with respect to the pyrano ring, as can be seen from the dihedral angle [89.83 $\left.(2)^{\circ}\right]$. The sum of the angles at atom N1 of the indolin-2-one moiety is in accordance with $s p^{2}$-hybridization $\left[359.41(2)^{\circ}\right]$.

The N1-H1 $\cdots \mathrm{O} 5$ hydrogen bonds connect two centrosymmetrically related molecules and generate the graph set motif $R_{2}^{2}(14)$ (Bernstein et al., 1995). The centrosymmetric dimers are interconnected into zig-zag linear chain of $\mathrm{C}-\mathrm{H} \cdots \mathrm{O}$ hydrogen bonds and the molecules form a layered structure (Fig. 2). In addition, there is a weak $\mathrm{C}-\mathrm{H} \cdots \pi$ interaction, viz. $\mathrm{C} 25-\mathrm{H} 25 \cdots \mathrm{Cg} 1(\mathrm{Cg} 1$ is the centroid of the ring C41-C46; symmetry codes are given in Table 1).

\section{S2. Experimental}

A mixture of 5-fluoroindoline-2,3-dione $(0.100 \mathrm{~g}, 0.60 \mathrm{mmol})$, 4-hydroxy- $2 H$-chromen-2-one $(0.194 \mathrm{~g}, 1.20 \mathrm{mmol})$, and paratoluene sulfonic acid $(0.114 \mathrm{~g}, 0.60 \mathrm{mmol})$ were dissolved in $5 \mathrm{ml}$ of ethanol:water $(1: 1 \mathrm{v} / \mathrm{v})$ and refluxed for $2 \mathrm{~h}$. After completion of the reaction as evident from TLC, the precipitated solid was filtered and washed with water to afford the product which was recrystallized from ethanol to provide colourless crystals. Yield 72\%, m.p. 541-543 K.

\section{S3. Refinement}

The N1-H atom was located in a difference map and refined with an $\mathrm{N}-\mathrm{H}$ distance restraint of $0.86 \pm 0.01 \AA$. The Cbound $\mathrm{H}$ atoms were placed at calculated positions and allowed to ride on their carrier atoms with $\mathrm{C}-\mathrm{H}=0.93 \AA$,and with $U_{\text {iso }}=1.2 U_{\text {eq }}(\mathrm{C})$. 


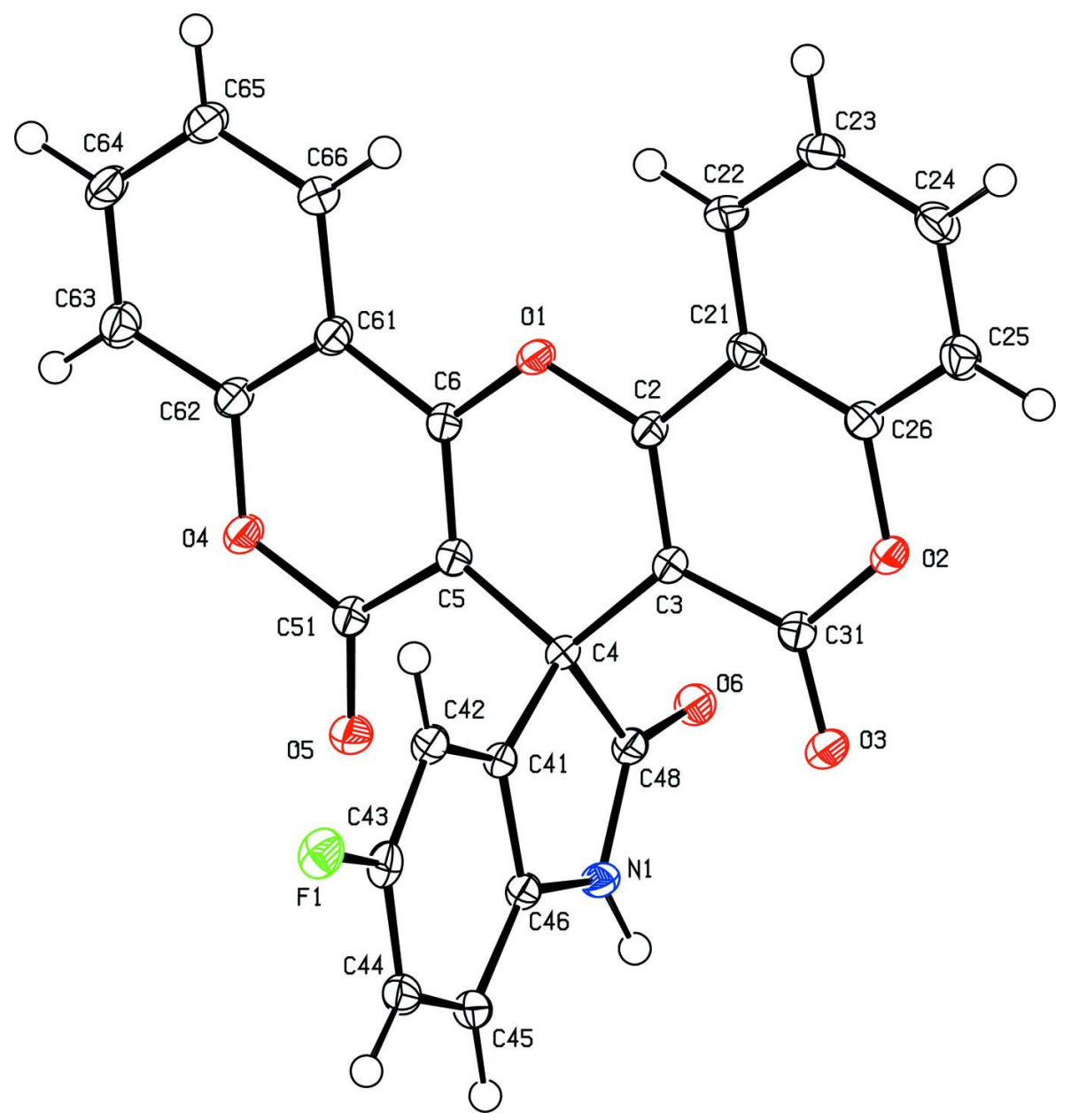

\section{Figure 1}

The molecular structure of (I), showing 50\% probability displacement ellipsoids and the atom-numbering scheme.

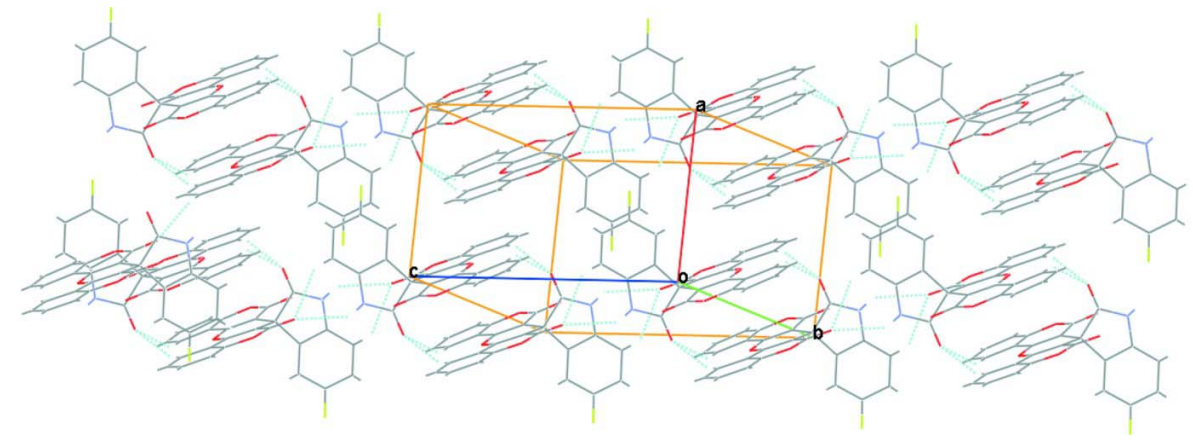

Figure 2

A packing diagram for (I).

5-Fluoro-6' $H, 7^{\prime} H, 8^{\prime} H$-spiro[indoline-3,7'- pyrano[3,2-c:5,6-c']di-1-benzopyran]-2,6', 8' $^{\prime}$-trione

Crystal data

$\mathrm{C}_{26} \mathrm{H}_{12} \mathrm{FNO}_{6}$

$M_{r}=453.37$

Triclinic, $P \overline{1}$

Hall symbol: -P 1 


$$
\begin{aligned}
& a=7.8262(1) \AA \\
& b=10.9278(1) \AA \\
& c=12.4067(2) \AA \\
& \alpha=113.374(1)^{\circ} \\
& \beta=94.922(1)^{\circ} \\
& \gamma=100.295(1)^{\circ} \\
& V=943.77(2) \AA^{3} \\
& Z=2 \\
& F(000)=464
\end{aligned}
$$

\section{Data collection}

\section{Bruker Kappa APEXII} diffractometer

Radiation source: fine-focus sealed tube Graphite monochromator Detector resolution: 0 pixels $\mathrm{mm}^{-1}$ $\omega$ and $\varphi$ scans

Absorption correction: multi-scan (SADABS; Sheldrick, 1996)

$$
T_{\min }=0.973, T_{\max }=0.978
$$

\section{Refinement}

Refinement on $F^{2}$

Least-squares matrix: full

$R\left[F^{2}>2 \sigma\left(F^{2}\right)\right]=0.045$

$w R\left(F^{2}\right)=0.124$

$S=1.04$

5504 reflections

311 parameters

1 restraint

Primary atom site location: structure-invariant direct methods
$D_{\mathrm{x}}=1.595 \mathrm{Mg} \mathrm{m}^{-3}$

Mo $K \alpha$ radiation, $\lambda=0.71073 \AA$

Cell parameters from 2000 reflections

$\theta=2-31^{\circ}$

$\mu=0.12 \mathrm{~mm}^{-1}$

$T=293 \mathrm{~K}$

Block, colourless

$0.23 \times 0.21 \times 0.18 \mathrm{~mm}$

15720 measured reflections

5504 independent reflections

4486 reflections with $I>2 \sigma(I)$

$R_{\text {int }}=0.023$

$\theta_{\text {max }}=30.1^{\circ}, \theta_{\min }=1.8^{\circ}$

$h=-11 \rightarrow 11$

$k=-15 \rightarrow 15$

$l=-17 \rightarrow 12$

Secondary atom site location: difference Fourier map

Hydrogen site location: inferred from neighbouring sites

$\mathrm{H}$ atoms treated by a mixture of independent and constrained refinement

$w=1 /\left[\sigma^{2}\left(F_{\mathrm{o}}^{2}\right)+(0.0602 P)^{2}+0.4439 P\right]$ where $P=\left(F_{\mathrm{o}}^{2}+2 F_{\mathrm{c}}^{2}\right) / 3$

$(\Delta / \sigma)_{\max }<0.001$

$\Delta \rho_{\max }=0.46$ e $\AA^{-3}$

$\Delta \rho_{\min }=-0.29$ e $\AA^{-3}$

\section{Special details}

Geometry. All e.s.d.'s (except the e.s.d. in the dihedral angle between two 1.s. planes) are estimated using the full covariance matrix. The cell e.s.d.'s are taken into account individually in the estimation of e.s.d.'s in distances, angles and torsion angles; correlations between e.s.d.'s in cell parameters are only used when they are defined by crystal symmetry. An approximate (isotropic) treatment of cell e.s.d.'s is used for estimating e.s.d.'s involving 1.s. planes.

Refinement. Refinement of $F^{2}$ against ALL reflections. The weighted $R$-factor $w R$ and goodness of fit $S$ are based on $F^{2}$, conventional $R$-factors $R$ are based on $F$, with $F$ set to zero for negative $F^{2}$. The threshold expression of $F^{2}>\sigma\left(F^{2}\right)$ is used only for calculating $R$-factors(gt) etc. and is not relevant to the choice of reflections for refinement. $R$-factors based on $F^{2}$ are statistically about twice as large as those based on $F$, and $R$-factors based on ALL data will be even larger.

Fractional atomic coordinates and isotropic or equivalent isotropic displacement parameters $\left(\AA^{2}\right)$

\begin{tabular}{lllll}
\hline & $x$ & $y$ & $z$ & $U_{\text {iso }} * / U_{\text {eq }}$ \\
\hline H1 & $-0.122(2)$ & $0.3850(19)$ & $0.4085(15)$ & $0.025(5)^{*}$ \\
C2 & $0.12458(17)$ & $0.34311(13)$ & $-0.00317(11)$ & $0.0140(2)$ \\
C3 & $0.05623(17)$ & $0.31770(13)$ & $0.08465(11)$ & $0.0135(2)$ \\
C4 & $0.09926(16)$ & $0.42071(13)$ & $0.21373(11)$ & $0.0128(2)$ \\
C5 & $0.18729(17)$ & $0.55860(13)$ & $0.21847(11)$ & $0.0139(2)$ \\
C6 & $0.24834(17)$ & $0.57319(13)$ & $0.12440(11)$ & $0.0139(2)$ \\
C21 & $0.09810(17)$ & $0.23914(13)$ & $-0.12381(11)$ & $0.0148(2)$
\end{tabular}




$\begin{array}{lllll}\text { C22 } & 0.17434(19) & 0.25796(14) & -0.21611(12) & 0.0172(3) \\ \text { H22 } & 0.2407 & 0.3436 & -0.2034 & 0.021^{*} \\ \text { C23 } & 0.15001(19) & 0.14852(15) & -0.32576(12) & 0.0191(3) \\ \text { H23 } & 0.2016 & 0.1604 & -0.3868 & 0.023^{*} \\ \text { C24 } & 0.0486(2) & 0.01981(15) & -0.34611(12) & 0.0199(3) \\ \text { H24 } & 0.0344 & -0.0533 & -0.4203 & 0.024^{*} \\ \text { C25 } & -0.03060(19) & 0.00031(14) & -0.25711(12) & 0.0194(3) \\ \text { H25 } & -0.1002 & -0.0847 & -0.2711 & 0.023^{*} \\ \text { C26 } & -0.00412(17) & 0.11020(14) & -0.14632(12) & 0.0156(3) \\ \text { C31 } & -0.05619(17) & 0.18354(14) & 0.05589(12) & 0.0152(2) \\ \text { C41 } & 0.21280(17) & 0.37727(13) & 0.29163(11) & 0.0130(2) \\ \text { C42 } & 0.37794(17) & 0.34883(13) & 0.28347(12) & 0.0155(3) \\ \text { H42 } & 0.4387 & 0.3545 & 0.2238 & 0.019^{*} \\ \text { C43 } & 0.44766(17) & 0.31133(14) & 0.36932(12) & 0.0164(3) \\ \text { C44 } & 0.36544(18) & 0.30651(14) & 0.46197(12) & 0.0168(3) \\ \text { H44 } & 0.4203 & 0.2841 & 0.5188 & 0.020^{*} \\ \text { C45 } & 0.19881(18) & 0.33555(13) & 0.46972(12) & 0.0156(3) \\ \text { H45 } & 0.1405 & 0.3334 & 0.5314 & 0.019^{*} \\ \text { C46 } & 0.12383(17) & 0.36764(13) & 0.38189(11) & 0.0134(2) \\ \text { C48 } & -0.07059(17) & 0.42709(13) & 0.27287(11) & 0.0140(2) \\ \text { C51 } & 0.21637(17) & 0.67890(13) & 0.33197(12) & 0.0157(3) \\ \text { C61 } & 0.34501(17) & 0.70292(14) & 0.13272(12) & 0.0151(2) \\ \text { C62 } & 0.37581(17) & 0.81431(14) & 0.24372(12) & 0.0162(3) \\ \text { C63 } & 0.47226(19) & 0.94335(14) & 0.26308(13) & 0.0196(3) \\ \text { H63 } & 0.4916 & 1.0166 & 0.3377 & 0.024^{*} \\ \text { C64 } & 0.53871(19) & 0.96018(15) & 0.16875(13) & 0.0198(3) \\ \text { H64 } & 0.6045 & 1.0455 & 0.1803 & 0.024^{*} \\ \text { C65 } & 0.50796(19) & 0.85014(15) & 0.05598(13) & 0.0195(3) \\ \text { H65 } & 0.5525 & 0.8632 & -0.0068 & 0.023^{*} \\ \text { C66 } & 0.41184(18) & 0.72236(14) & 0.03748(12) & 0.0173(3) \\ \text { H66 } & 0.3914 & 0.6496 & -0.0375 & 0.021^{*} \\ \text { N1 } & -0.04170(15) & 0.39656(12) & 0.36839(10) & 0.0140(2) \\ \text { O1 } & 0.22685(13) & 0.46733(10) & 0.01416(8) & 0.0159(2) \\ \text { O2 } & -0.08257(13) & 0.08560(10) & -0.05945(8) & 0.0167(2) \\ \text { O3 } & -0.12795(14) & 0.15262(10) & 0.12640(9) & 0.0195(2) \\ \text { O4 } & 0.31252(13) & 0.80145(10) & 0.33995(9) & 0.0180(2) \\ \text { O5 } & 0.16406(14) & 0.67882(10) & 0.42086(9) & 0.0194(2) \\ \text { O6 } & -0.20150(13) & 0.45589(10) & 0.23875(9) & 0.0168(2) \\ \text { F1 } & 0.60614(11) & 0.27654(9) & 0.36098(8) & 0.02124(19) \\ & & & & \end{array}$

Atomic displacement parameters $\left(A^{2}\right)$

\begin{tabular}{lllllll}
\hline & $U^{11}$ & $U^{22}$ & $U^{33}$ & $U^{12}$ & $U^{13}$ & $U^{23}$ \\
\hline C2 & $0.0134(6)$ & $0.0131(6)$ & $0.0156(6)$ & $0.0008(4)$ & $0.0019(4)$ & $0.0072(5)$ \\
C3 & $0.0134(6)$ & $0.0124(6)$ & $0.0142(6)$ & $0.0009(4)$ & $0.0018(4)$ & $0.0060(5)$ \\
C4 & $0.0124(5)$ & $0.0129(6)$ & $0.0132(5)$ & $0.0006(4)$ & $0.0022(4)$ & $0.0066(5)$ \\
C5 & $0.0134(6)$ & $0.0135(6)$ & $0.0142(6)$ & $0.0013(4)$ & $0.0013(4)$ & $0.0062(5)$ \\
C6 & $0.0128(6)$ & $0.0130(6)$ & $0.0145(6)$ & $0.0012(4)$ & $0.0013(4)$ & $0.0054(5)$
\end{tabular}




$\begin{array}{lllllll}\text { C21 } & 0.0159(6) & 0.0150(6) & 0.0139(6) & 0.0036(5) & 0.0018(4) & 0.0065(5) \\ \text { C22 } & 0.0205(6) & 0.0170(6) & 0.0165(6) & 0.0032(5) & 0.0028(5) & 0.0101(5) \\ \text { C23 } & 0.0233(7) & 0.0214(7) & 0.0144(6) & 0.0047(5) & 0.0043(5) & 0.0095(5) \\ \text { C24 } & 0.0264(7) & 0.0169(6) & 0.0139(6) & 0.0040(5) & 0.0003(5) & 0.0051(5) \\ \text { C25 } & 0.0226(7) & 0.0147(6) & 0.0178(6) & 0.0015(5) & -0.0009(5) & 0.0059(5) \\ \text { C26 } & 0.0146(6) & 0.0167(6) & 0.0157(6) & 0.0021(5) & 0.0014(5) & 0.0080(5) \\ \text { C31 } & 0.0144(6) & 0.0151(6) & 0.0157(6) & 0.0023(5) & 0.0017(4) & 0.0067(5) \\ \text { C41 } & 0.0142(6) & 0.0113(5) & 0.0122(5) & 0.0000(4) & 0.0009(4) & 0.0050(4) \\ \text { C42 } & 0.0146(6) & 0.0147(6) & 0.0164(6) & 0.0010(5) & 0.0029(5) & 0.0066(5) \\ \text { C43 } & 0.0117(6) & 0.0148(6) & 0.0209(6) & 0.0014(5) & 0.0009(5) & 0.0068(5) \\ \text { C44 } & 0.0172(6) & 0.0157(6) & 0.0173(6) & 0.0017(5) & -0.0010(5) & 0.0085(5) \\ \text { C45 } & 0.0176(6) & 0.0142(6) & 0.0138(6) & 0.0017(5) & 0.0019(5) & 0.0058(5) \\ \text { C46 } & 0.0136(6) & 0.0125(6) & 0.0132(5) & 0.0013(4) & 0.0025(4) & 0.0051(4) \\ \text { C48 } & 0.0147(6) & 0.0125(6) & 0.0138(5) & 0.0007(4) & 0.0026(4) & 0.0055(5) \\ \text { C51 } & 0.0150(6) & 0.0140(6) & 0.0166(6) & 0.0003(5) & 0.0010(5) & 0.0066(5) \\ \text { C61 } & 0.0136(6) & 0.0153(6) & 0.0173(6) & 0.0017(5) & 0.0016(5) & 0.0086(5) \\ \text { C62 } & 0.0150(6) & 0.0157(6) & 0.0183(6) & 0.0012(5) & 0.0032(5) & 0.0084(5) \\ \text { C63 } & 0.0195(6) & 0.0159(6) & 0.0209(6) & 0.0011(5) & 0.0029(5) & 0.0066(5) \\ \text { C64 } & 0.0192(6) & 0.0163(6) & 0.0256(7) & 0.0000(5) & 0.0042(5) & 0.0122(6) \\ \text { C65 } & 0.0180(6) & 0.0204(7) & 0.0227(7) & 0.0018(5) & 0.0048(5) & 0.0126(6) \\ \text { C66 } & 0.0181(6) & 0.0175(6) & 0.0170(6) & 0.0018(5) & 0.0029(5) & 0.0090(5) \\ \text { N1 } & 0.0130(5) & 0.0165(5) & 0.0142(5) & 0.0029(4) & 0.0043(4) & 0.0080(4) \\ \text { O1 } & 0.0200(5) & 0.0127(4) & 0.0133(4) & -0.0008(4) & 0.0042(3) & 0.0055(4) \\ \text { O2 } & 0.0186(5) & 0.0141(4) & 0.0153(4) & -0.0010(4) & 0.0031(4) & 0.0062(4) \\ \text { O3 } & 0.0216(5) & 0.0177(5) & 0.0188(5) & -0.0001(4) & 0.0049(4) & 0.0090(4) \\ \text { O4 } & 0.0216(5) & 0.0141(4) & 0.0157(4) & -0.0015(4) & 0.0047(4) & 0.0055(4) \\ \text { O5 } & 0.0223(5) & 0.0189(5) & 0.0149(4) & 0.0007(4) & 0.0045(4) & 0.0063(4) \\ \text { O6 } & 0.0150(4) & 0.0181(5) & 0.0186(5) & 0.0037(4) & 0.0022(3) & 0.0092(4) \\ \text { F1 } & 0.0125(4) & 0.0252(4) & 0.0288(5) & 0.0054(3) & 0.0035(3) & 0.0138(4)\end{array}$

Geometric parameters $\left(\AA,{ }^{o}\right)$

\begin{tabular}{llll}
\hline $\mathrm{C} 2-\mathrm{C} 3$ & $1.3555(18)$ & $\mathrm{C} 41-\mathrm{C} 46$ & $1.3968(18)$ \\
$\mathrm{C} 2-\mathrm{O} 1$ & $1.3712(16)$ & $\mathrm{C} 42-\mathrm{C} 43$ & $1.3880(18)$ \\
$\mathrm{C} 2-\mathrm{C} 21$ & $1.4448(18)$ & $\mathrm{C} 42-\mathrm{H} 42$ & 0.9300 \\
$\mathrm{C} 3-\mathrm{C} 31$ & $1.4563(18)$ & $\mathrm{C} 43-\mathrm{F} 1$ & $1.3612(15)$ \\
$\mathrm{C} 3-\mathrm{C} 4$ & $1.5122(17)$ & $\mathrm{C} 43-\mathrm{C} 44$ & $1.379(2)$ \\
$\mathrm{C} 4-\mathrm{C} 5$ & $1.5167(18)$ & $\mathrm{C} 44-\mathrm{C} 45$ & $1.3985(19)$ \\
$\mathrm{C} 4-\mathrm{C} 41$ & $1.5219(17)$ & $\mathrm{C} 44-\mathrm{H} 44$ & 0.9300 \\
$\mathrm{C} 4-\mathrm{C} 48$ & $1.5716(18)$ & $\mathrm{C} 45-\mathrm{C} 46$ & $1.3862(18)$ \\
$\mathrm{C} 5-\mathrm{C} 6$ & $1.3535(18)$ & $\mathrm{C} 45-\mathrm{H} 45$ & 0.9300 \\
$\mathrm{C} 5-\mathrm{C} 51$ & $1.4602(18)$ & $\mathrm{C} 46-\mathrm{N} 1$ & $1.3990(16)$ \\
$\mathrm{C} 6-\mathrm{O} 1$ & $1.3685(16)$ & $\mathrm{C} 48-\mathrm{O} 6$ & $1.2144(16)$ \\
$\mathrm{C} 6-\mathrm{C} 61$ & $1.4423(18)$ & $\mathrm{C} 48-\mathrm{N} 1$ & $1.3643(17)$ \\
$\mathrm{C} 21-\mathrm{C} 26$ & $1.3963(19)$ & $\mathrm{C} 51-\mathrm{O} 5$ & $1.2091(17)$ \\
$\mathrm{C} 21-\mathrm{C} 22$ & $1.4036(19)$ & $\mathrm{C} 51-\mathrm{O} 4$ & $1.3753(16)$ \\
$\mathrm{C} 22-\mathrm{C} 23$ & $1.3785(19)$ & $\mathrm{C} 61-\mathrm{C} 62$ & $1.4074(19)$ \\
$\mathrm{C} 22-\mathrm{H} 22$ & 0.9300 & $\mathrm{C} 61-\mathrm{C} 66$ &
\end{tabular}




\begin{tabular}{|c|c|c|c|}
\hline $\mathrm{C} 23-\mathrm{C} 24$ & $1.399(2)$ & $\mathrm{C} 62-\mathrm{O} 4$ & $1.3785(16)$ \\
\hline $\mathrm{C} 23-\mathrm{H} 23$ & 0.9300 & $\mathrm{C} 62-\mathrm{C} 63$ & $1.3913(19)$ \\
\hline $\mathrm{C} 24-\mathrm{C} 25$ & $1.381(2)$ & $\mathrm{C} 63-\mathrm{C} 64$ & $1.384(2)$ \\
\hline $\mathrm{C} 24-\mathrm{H} 24$ & 0.9300 & C63-H63 & 0.9300 \\
\hline $\mathrm{C} 25-\mathrm{C} 26$ & $1.3881(19)$ & $\mathrm{C} 64-\mathrm{C} 65$ & $1.402(2)$ \\
\hline $\mathrm{C} 25-\mathrm{H} 25$ & 0.9300 & C64-H64 & 0.9300 \\
\hline $\mathrm{C} 26-\mathrm{O} 2$ & $1.3767(16)$ & $\mathrm{C} 65-\mathrm{C} 66$ & $1.3817(19)$ \\
\hline $\mathrm{C} 31-\mathrm{O} 3$ & $1.2025(17)$ & C65-H65 & 0.9300 \\
\hline $\mathrm{C} 31-\mathrm{O} 2$ & $1.3751(16)$ & C66-H66 & 0.9300 \\
\hline $\mathrm{C} 41-\mathrm{C} 42$ & $1.3851(18)$ & $\mathrm{N} 1-\mathrm{H} 1$ & $0.855(9)$ \\
\hline $\mathrm{C} 3-\mathrm{C} 2-\mathrm{O} 1$ & $123.63(12)$ & $\mathrm{C} 43-\mathrm{C} 42-\mathrm{H} 42$ & 121.9 \\
\hline $\mathrm{C} 3-\mathrm{C} 2-\mathrm{C} 21$ & $122.30(12)$ & $\mathrm{F} 1-\mathrm{C} 43-\mathrm{C} 44$ & $118.16(12)$ \\
\hline $\mathrm{O} 1-\mathrm{C} 2-\mathrm{C} 21$ & $114.06(11)$ & $\mathrm{F} 1-\mathrm{C} 43-\mathrm{C} 42$ & $117.95(12)$ \\
\hline $\mathrm{C} 2-\mathrm{C} 3-\mathrm{C} 31$ & $119.28(12)$ & $\mathrm{C} 44-\mathrm{C} 43-\mathrm{C} 42$ & $123.88(12)$ \\
\hline $\mathrm{C} 2-\mathrm{C} 3-\mathrm{C} 4$ & $122.74(11)$ & $\mathrm{C} 43-\mathrm{C} 44-\mathrm{C} 45$ & $119.46(12)$ \\
\hline $\mathrm{C} 31-\mathrm{C} 3-\mathrm{C} 4$ & $117.91(11)$ & $\mathrm{C} 43-\mathrm{C} 44-\mathrm{H} 44$ & 120.3 \\
\hline $\mathrm{C} 3-\mathrm{C} 4-\mathrm{C} 5$ & $108.11(10)$ & $\mathrm{C} 45-\mathrm{C} 44-\mathrm{H} 44$ & 120.3 \\
\hline $\mathrm{C} 3-\mathrm{C} 4-\mathrm{C} 41$ & $112.55(10)$ & $\mathrm{C} 46-\mathrm{C} 45-\mathrm{C} 44$ & $117.45(12)$ \\
\hline $\mathrm{C} 5-\mathrm{C} 4-\mathrm{C} 41$ & $111.75(10)$ & $\mathrm{C} 46-\mathrm{C} 45-\mathrm{H} 45$ & 121.3 \\
\hline $\mathrm{C} 3-\mathrm{C} 4-\mathrm{C} 48$ & $111.18(10)$ & $\mathrm{C} 44-\mathrm{C} 45-\mathrm{H} 45$ & 121.3 \\
\hline $\mathrm{C} 5-\mathrm{C} 4-\mathrm{C} 48$ & $112.06(10)$ & $\mathrm{C} 45-\mathrm{C} 46-\mathrm{C} 41$ & $122.00(12)$ \\
\hline $\mathrm{C} 41-\mathrm{C} 4-\mathrm{C} 48$ & $101.17(10)$ & $\mathrm{C} 45-\mathrm{C} 46-\mathrm{N} 1$ & $128.16(12)$ \\
\hline $\mathrm{C} 6-\mathrm{C} 5-\mathrm{C} 51$ & $119.03(12)$ & $\mathrm{C} 41-\mathrm{C} 46-\mathrm{N} 1$ & $109.83(11)$ \\
\hline $\mathrm{C} 6-\mathrm{C} 5-\mathrm{C} 4$ & $122.84(12)$ & $\mathrm{O} 6-\mathrm{C} 48-\mathrm{N} 1$ & $127.29(13)$ \\
\hline $\mathrm{C} 51-\mathrm{C} 5-\mathrm{C} 4$ & $118.03(11)$ & $\mathrm{O} 6-\mathrm{C} 48-\mathrm{C} 4$ & $125.09(12)$ \\
\hline $\mathrm{C} 5-\mathrm{C} 6-\mathrm{O} 1$ & $123.61(12)$ & $\mathrm{N} 1-\mathrm{C} 48-\mathrm{C} 4$ & $107.61(11)$ \\
\hline $\mathrm{C} 5-\mathrm{C} 6-\mathrm{C} 61$ & $122.40(12)$ & $\mathrm{O} 5-\mathrm{C} 51-\mathrm{O} 4$ & $117.02(12)$ \\
\hline $\mathrm{O} 1-\mathrm{C} 6-\mathrm{C} 61$ & $113.99(11)$ & $\mathrm{O} 5-\mathrm{C} 51-\mathrm{C} 5$ & $124.82(12)$ \\
\hline $\mathrm{C} 26-\mathrm{C} 21-\mathrm{C} 22$ & $118.97(12)$ & $\mathrm{O} 4-\mathrm{C} 51-\mathrm{C} 5$ & $118.15(12)$ \\
\hline $\mathrm{C} 26-\mathrm{C} 21-\mathrm{C} 2$ & $116.49(12)$ & C62-C61-C66 & $118.90(12)$ \\
\hline $\mathrm{C} 22-\mathrm{C} 21-\mathrm{C} 2$ & $124.47(12)$ & $\mathrm{C} 62-\mathrm{C} 61-\mathrm{C} 6$ & $116.85(12)$ \\
\hline $\mathrm{C} 23-\mathrm{C} 22-\mathrm{C} 21$ & $119.45(13)$ & $\mathrm{C} 66-\mathrm{C} 61-\mathrm{C} 6$ & $124.23(12)$ \\
\hline $\mathrm{C} 23-\mathrm{C} 22-\mathrm{H} 22$ & 120.3 & $\mathrm{O} 4-\mathrm{C} 62-\mathrm{C} 63$ & $116.74(12)$ \\
\hline $\mathrm{C} 21-\mathrm{C} 22-\mathrm{H} 22$ & 120.3 & $\mathrm{O} 4-\mathrm{C} 62-\mathrm{C} 61$ & $121.41(12)$ \\
\hline $\mathrm{C} 22-\mathrm{C} 23-\mathrm{C} 24$ & 120.67 (13) & $\mathrm{C} 63-\mathrm{C} 62-\mathrm{C} 61$ & $121.85(13)$ \\
\hline $\mathrm{C} 22-\mathrm{C} 23-\mathrm{H} 23$ & 119.7 & $\mathrm{C} 64-\mathrm{C} 63-\mathrm{C} 62$ & $118.45(13)$ \\
\hline $\mathrm{C} 24-\mathrm{C} 23-\mathrm{H} 23$ & 119.7 & $\mathrm{C} 64-\mathrm{C} 63-\mathrm{H} 63$ & 120.8 \\
\hline $\mathrm{C} 25-\mathrm{C} 24-\mathrm{C} 23$ & $120.62(13)$ & С62-C63-H63 & 120.8 \\
\hline $\mathrm{C} 25-\mathrm{C} 24-\mathrm{H} 24$ & 119.7 & $\mathrm{C} 63-\mathrm{C} 64-\mathrm{C} 65$ & $120.78(13)$ \\
\hline $\mathrm{C} 23-\mathrm{C} 24-\mathrm{H} 24$ & 119.7 & C63-C64-H64 & 119.6 \\
\hline $\mathrm{C} 24-\mathrm{C} 25-\mathrm{C} 26$ & $118.62(13)$ & $\mathrm{C} 65-\mathrm{C} 64-\mathrm{H} 64$ & 119.6 \\
\hline $\mathrm{C} 24-\mathrm{C} 25-\mathrm{H} 25$ & 120.7 & C66-C65-C64 & $120.39(13)$ \\
\hline $\mathrm{C} 26-\mathrm{C} 25-\mathrm{H} 25$ & 120.7 & C66-C65-H65 & 119.8 \\
\hline $\mathrm{O} 2-\mathrm{C} 26-\mathrm{C} 25$ & $116.72(12)$ & C64-C65-H65 & 119.8 \\
\hline $\mathrm{O} 2-\mathrm{C} 26-\mathrm{C} 21$ & $121.62(12)$ & $\mathrm{C} 65-\mathrm{C} 66-\mathrm{C} 61$ & $119.62(13)$ \\
\hline $\mathrm{C} 25-\mathrm{C} 26-\mathrm{C} 21$ & $121.66(13)$ & $\mathrm{C} 65-\mathrm{C} 66-\mathrm{H} 66$ & 120.2 \\
\hline $\mathrm{O} 3-\mathrm{C} 31-\mathrm{O} 2$ & $117.67(12)$ & C61-C66-H66 & 120.2 \\
\hline
\end{tabular}




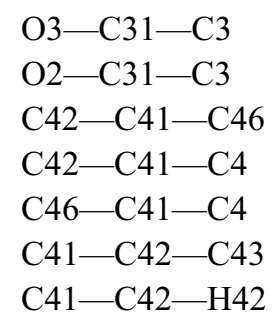

$124.30(12)$

$118.03(12)$

$120.82(12)$

$130.10(12)$

$109.08(11)$

$116.28(12)$

121.9

$177.76(11)$

$-3.55(19)$

$-5.5(2)$

$173.14(11)$

$13.71(17)$

$-169.55(11)$

$-110.20(14)$

$66.54(15)$

$137.11(13)$

$-46.15(15)$

$-13.18(17)$

$111.21(14)$

-136.05 (13)

$170.66(11)$

$-64.95(15)$

47.79 (15)

-179.49 (11)

$4.4(2)$

1.49 (19)

$-174.63(11)$

$0.75(19)$

$179.55(11)$

$-176.13(12)$

$2.68(18)$

$-1.41(19)$

$175.39(12)$

$0.8(2)$

$0.7(2)$

$-1.5(2)$

$-178.75(12)$

$0.8(2)$

$-179.85(11)$

$3.10(18)$

$0.6(2)$

$-176.44(12)$

$-177.26(13)$

$5.88(19)$

$2.63(18)$

$-174.23(11)$

$58.76(18)$

$$
\begin{aligned}
& \mathrm{C} 48-\mathrm{N} 1-\mathrm{C} 46 \\
& \mathrm{C} 48-\mathrm{N} 1-\mathrm{H} 1 \\
& \mathrm{C} 46-\mathrm{N} 1-\mathrm{H} 1 \\
& \mathrm{C} 6-\mathrm{O} 1-\mathrm{C} 2 \\
& \mathrm{C} 31-\mathrm{O} 2-\mathrm{C} 26 \\
& \mathrm{C} 51-\mathrm{O} 4-\mathrm{C} 62
\end{aligned}
$$

$\mathrm{C} 42-\mathrm{C} 43-\mathrm{C} 44-\mathrm{C} 45$

$\mathrm{C} 43-\mathrm{C} 44-\mathrm{C} 45-\mathrm{C} 46$

$\mathrm{C} 44-\mathrm{C} 45-\mathrm{C} 46-\mathrm{C} 41$

$\mathrm{C} 44-\mathrm{C} 45-\mathrm{C} 46-\mathrm{N} 1$

$\mathrm{C} 42-\mathrm{C} 41-\mathrm{C} 46-\mathrm{C} 45$

$\mathrm{C} 4-\mathrm{C} 41-\mathrm{C} 46-\mathrm{C} 45$

$\mathrm{C} 42-\mathrm{C} 41-\mathrm{C} 46-\mathrm{N} 1$

$\mathrm{C} 4-\mathrm{C} 41-\mathrm{C} 46-\mathrm{N} 1$

$\mathrm{C} 3-\mathrm{C} 4-\mathrm{C} 48-\mathrm{O} 6$

$\mathrm{C} 5-\mathrm{C} 4-\mathrm{C} 48-\mathrm{O} 6$

$\mathrm{C} 41-\mathrm{C} 4-\mathrm{C} 48-\mathrm{O} 6$

$\mathrm{C} 3-\mathrm{C} 4-\mathrm{C} 48-\mathrm{N} 1$

$\mathrm{C} 5-\mathrm{C} 4-\mathrm{C} 48-\mathrm{N} 1$

$\mathrm{C} 41-\mathrm{C} 4-\mathrm{C} 48-\mathrm{N} 1$

$\mathrm{C} 6-\mathrm{C} 5-\mathrm{C} 51-\mathrm{O} 5$

$\mathrm{C} 4-\mathrm{C} 5-\mathrm{C} 51-\mathrm{O} 5$

$\mathrm{C} 6-\mathrm{C} 5-\mathrm{C} 51-\mathrm{O} 4$

$\mathrm{C} 4-\mathrm{C} 5-\mathrm{C} 51-\mathrm{O} 4$

$\mathrm{C} 5-\mathrm{C} 6-\mathrm{C} 61-\mathrm{C} 62$

$\mathrm{O} 1-\mathrm{C} 6-\mathrm{C} 61-\mathrm{C} 62$

C5-C6-C61-C66

$\mathrm{O} 1-\mathrm{C} 6-\mathrm{C} 61-\mathrm{C} 66$

C66-C61-C62-O4

C6-C61-C62-O4

C66-C61-C62-C63

C6-C61-C62-C63

$\mathrm{O} 4-\mathrm{C} 62-\mathrm{C} 63-\mathrm{C} 64$

C61-C62-C63-C64

C62-C63-C64-C65

C63-C64-C65-C66

C64-C65-C66-C61

C62-C61-C66-C65

C6-C61-C66-C65

$\mathrm{O} 6-\mathrm{C} 48-\mathrm{N} 1-\mathrm{C} 46$

$\mathrm{C} 4-\mathrm{C} 48-\mathrm{N} 1-\mathrm{C} 46$

$\mathrm{C} 45-\mathrm{C} 46-\mathrm{N} 1-\mathrm{C} 48$

$\mathrm{C} 41-\mathrm{C} 46-\mathrm{N} 1-\mathrm{C} 48$

$\mathrm{C} 5-\mathrm{C} 6-\mathrm{O} 1-\mathrm{C} 2$

$\mathrm{C} 61-\mathrm{C} 6-\mathrm{O} 1-\mathrm{C} 2$

$\mathrm{C} 3-\mathrm{C} 2-\mathrm{O} 1-\mathrm{C} 6$
112.25 (11)

$123.1(13)$

124.1 (13)

$117.29(10)$

$122.11(11)$

122.08 (11)

2.5 (2)

0.25 (19)

$-2.90(19)$

$178.11(12)$

$2.91(19)$

$-177.21(12)$

$-177.93(11)$

$1.94(14)$

$-59.04(17)$

62.07 (16)

$-178.75(12)$

$121.75(11)$

-117.14 (11)

2.04 (13)

$178.02(13)$

$-5.7(2)$

$-3.06(18)$

$173.26(11)$

0.86 (19)

$-178.25(11)$

$179.52(12)$

0.41 (19)

179.57 (12)

-1.69 (19)

-0.7 (2)

$177.99(12)$

$179.70(12)$

$0.0(2)$

0.7 (2)

$-0.6(2)$

$-0.2(2)$

0.8 (2)

$-177.82(12)$

$179.76(13)$

-1.05 (14)

178.55 (13)

-0.54 (15)

$5.83(18)$

$-175.07(11)$

$-5.26(18)$ 
supporting information

$\begin{array}{llll}\mathrm{C} 5-\mathrm{C} 4-\mathrm{C} 41-\mathrm{C} 42 & -63.11(17) & \mathrm{C} 21-\mathrm{C} 2-\mathrm{O} 1-\mathrm{C} 6 & 175.95(11) \\ \mathrm{C} 48-\mathrm{C} 4-\mathrm{C} 41-\mathrm{C} 42 & 177.49(13) & \mathrm{O} 3-\mathrm{C} 31-\mathrm{O} 2-\mathrm{C} 26 & -178.97(11) \\ \mathrm{C} 3-\mathrm{C} 4-\mathrm{C} 41-\mathrm{C} 46 & -121.10(12) & \mathrm{C} 3-\mathrm{C} 31-\mathrm{O} 2-\mathrm{C} 26 & 1.14(17) \\ \mathrm{C} 5-\mathrm{C} 4-\mathrm{C} 41-\mathrm{C} 46 & 117.03(12) & \mathrm{C} 25-\mathrm{C} 26-\mathrm{O} 2-\mathrm{C} 31 & 175.47(12) \\ \mathrm{C} 48-\mathrm{C} 4-\mathrm{C} 41-\mathrm{C} 46 & -2.37(13) & \mathrm{C} 21-\mathrm{C} 26-\mathrm{O} 2-\mathrm{C} 31 & -4.09(18) \\ \mathrm{C} 46-\mathrm{C} 41-\mathrm{C} 42-\mathrm{C} 43 & -0.17(18) & \mathrm{O} 5-\mathrm{C} 51-\mathrm{O} 4-\mathrm{C} 62 & -178.69(12) \\ \mathrm{C} 4-\mathrm{C} 41-\mathrm{C} 42-\mathrm{C} 43 & 179.98(12) & \mathrm{C} 5-\mathrm{C} 51-\mathrm{O} 4-\mathrm{C} 62 & 2.31(18) \\ \mathrm{C} 41-\mathrm{C} 42-\mathrm{C} 43-\mathrm{F} 1 & 177.06(11) & \mathrm{C} 63-\mathrm{C} 62-\mathrm{O} 4-\mathrm{C} 51 & -179.61(12) \\ \mathrm{C} 41-\mathrm{C} 42-\mathrm{C} 43-\mathrm{C} 44 & -2.6(2) & \mathrm{C} 61-\mathrm{C} 62-\mathrm{O} 4-\mathrm{C} 51 & 0.08(19) \\ \mathrm{F} 1-\mathrm{C} 43-\mathrm{C} 44-\mathrm{C} 45 & -177.07(11) & & \end{array}$

Hydrogen-bond geometry $\left(A,{ }^{\circ}\right)$

$\mathrm{Cg} 1$ is the centroid of the $\mathrm{C} 41-\mathrm{C} 46$ ring.

\begin{tabular}{lllll}
\hline$D-\mathrm{H} \cdots A$ & $D-\mathrm{H}$ & $\mathrm{H} \cdots A$ & $D \cdots A$ & $D-\mathrm{H}^{\cdots} A$ \\
\hline $\mathrm{C} 45-\mathrm{H} 45 \cdots \mathrm{O} 5^{\mathrm{i}}$ & 0.93 & 2.50 & $3.2564(18)$ & 139 \\
$\mathrm{C} 22-\mathrm{H} 22 \cdots 6^{\mathrm{ii}}$ & 0.93 & 2.46 & $3.2205(17)$ & 139 \\
$\mathrm{C} 66-\mathrm{H} 66 \cdots 6^{i i}$ & 0.93 & 2.50 & $3.2921(17)$ & 144 \\
$\mathrm{C} 64-\mathrm{H} 64 \cdots \mathrm{O} 3^{\mathrm{iii}}$ & 0.93 & 2.49 & $3.2641(18)$ & 141 \\
$\mathrm{C} 25-\mathrm{H} 25 \cdots C g 1^{\text {iv }}$ & 0.93 & 2.65 & $3.5482(17)$ & 163 \\
$\mathrm{~N} 1-\mathrm{H} 1 \cdots{ }^{\mathrm{v}}$ & $0.86(2)$ & $2.15(2)$ & $2.810(2)$ & $134(2)$ \\
$\mathrm{N} 1-\mathrm{H} 1 \cdots{ }^{\mathrm{v}}$ & $0.86(2)$ & $2.51(2)$ & $3.208(2)$ & $140(2)$
\end{tabular}

Symmetry codes: (i) $-x,-y+1,-z+1$; (ii) $-x,-y+1,-z$; (iii) $x+1, y+1, z$; (iv) $-x,-y,-z$; (v) $x-1, y, z$. 